\title{
Analysis Of Distance Measurement System Of Leading Vehicle
}

\author{
Ms. Priyanka D. Deshmukh ${ }^{1}$ and Prof. G.P.Dhok ${ }^{2}$ \\ ${ }^{1}$ Department of Electronics and Telecommunication, \\ Sipna's college of Engineering and Technology, Amravati \\ Email:piyudeshmukh20070rediffmail.com \\ ${ }^{2}$ Department of Electronics and Telecommunication \\ Sipna's college of Engineering and Technology, Amravati \\ Email:gajanandhokerediffmail.com
}

\begin{abstract}
Abtract
Based on the digital image processing theory, a new method of measuring the leading vehicle distance was proposed. The input image using the method of edge enhancement and morphological transformation was established, so the edges of objects were enhanced to identify. The target vehicle was identified and calibrated in the image by using the method of the obstacle detection by segmentation and decision tree. The relationship between coordinates value in image space and the data of the real space plane was established by applying the ray angles. Thus, through accessing to image pixel coordinates of the vehicle, the vehicle's actual position in the plane can be calculated. At last, the leading vehicle distance based on the calculating model of inverse perspective mapping was measured. By using software VC++, an experiment program was made. The experiment results prove that the method of measuring the leading vehicle distance is simple and effective. It can meet the requirement of intelligent vehicle technologies. It is an more available and more advanced method to calculate the leading vehicle distance. The target vehicle was identified and calibrated in the image by using the method of the obstacle detection by segmentation and decision tree. The relationship between coordinates value in image space and the data of the real space.
\end{abstract}

\section{Keywords}

Digital Image Processing, Obstacle Detection, Monocular Ranging, Active Safety, Leading Vehicle Distance.

\section{Introduction}

Advanced Vehicle Control System (AVCS) is an important component of the Intelligent Transportation System. By measuring the leading vehicle distance, it can provide effective vehicle driving information for the prevention of traffic accident, thereby enhancing traffic safety level. Based on domestic and international Vision Ranging technology and digital image processing theory research, this paper proposes a new method of measuring the leading vehicle 
distance based on the digital image processing theory, and obtains the leading vehicle distance data through the image pre-processing, obstacle detection and ranging model calculation and other steps. This method is a passive ranging method, and not transmits the signal to the outside environment, furthermore, also has easy to update algorithm, equipment light etc. [1].

In visual approach for distance measuring. Firstly, images are taken in different positions. Then the scaling parameter between these images could be calculated with the Fourier-Mellin transform. The Fourier-Mellin transform is successfully utilized for figuring out the scaling and rotation parameters between images, meanwhile the transform itself is translation independent. So when the images are rotated or translated, we can also get a correspondingly accurate scaling parameter. [2]

Visual data mining is one of important approach of data mining techniques. Most of them are based on computer graphic techniques but few of them exploit image-processing techniques. This paper proposes an image processing method, named RNAM (resemble neighborhood averaging method), to facilitate visual data mining, which is used to post-process the data mining resultimage and help users to discover significant features and useful patterns effectively[3].

\section{Image Pre-Processing Method of Choice}

Image pre-processing can improve image quality and reduce image noise and lay the foundation for obstacle detection. Required to select an appropriate way in digital image processing method to conduct a comprehensive pre-preprocessing to image, this paper selects edge enhancement and method of morphological opening and closing to smooth details and to enhance the edges of objects so as to identify.

\subsection{Edge Enhancement}

Because image edge depicts the contour, enhancing the image edges has important implications for the object recognition. Image enhancement is divided into two main categories: Space domain method and frequency domain method. Study the use of space-domain processing methods, as much as possible to shorten the processing. In the pixel matrix size of $\mathrm{M}$ rows, $\mathrm{N}$ columns of the image, the template $\mathrm{R}$ with $\mathrm{m}$ rows, $\mathrm{n}$ columns carried space domain linear filtering is given by the following formula:

$\mathrm{R}=w_{1} z_{1}+w_{2} z_{2}+----+w_{m n} z_{m n}=\sum_{i=1}^{m n} w_{i} z_{i}$

$w_{m n}$ is the template coefficient and $z_{m n}$ is the image pixels under template.

Using Laplace second-order differential image edge enhancement, the corresponding algorithm is as follows:

$g(x, y)=f(x, y)-\nabla f^{2}$

Where $f(x, y)$ is the image before handling;

$g(x, y)$ is the image after Laplace enhancement; and

define Laplace transform operator $\nabla f^{2}$ as:

$\nabla f^{2}=\frac{\partial^{2 f}}{\partial x^{2}}+\frac{\partial^{2 f}}{\partial y^{2}}$ 
Find that :

$\nabla f^{2}=f(x+1, y)+f(x-1, y)+f(x, y+1)+f(x, y-1)-4 f(x, y)$

Using Laplace transformation the image is much clearer

than before, with edge part enhancement and background keynote maintenance.

\subsection{Operation of Morphological Opening and Closing}

While after space domain enhancement, some interference factors in the image possibly impact Obstacle Detection. This paper focuses on image noise reduction by the operation of the mathematical morphological opening and closing. With the features of mathematical morphology operation, processing the image captured by grey scale open and close, the effects of image noise removal is the best[2]. Both of the calculations addressed above are the combination of the open calculation $(\mathrm{O})$ and the close calculation $(\mathrm{C})$, which can be represented as:

$O C=(A \circ B) \bullet(-B) \quad$ and

$C O=[A \bullet(-B)] \circ B$

A refers to the original image; and B refers to structure element. The open and close calculation not only make extracting the separate targets easier, but will eliminate some noises, making the grey scale in the target more even and then smoother. As a result, it can eliminate the artifact when extracting the outline of the target, improving the image quality.

\subsubsection{Morphology Fundamentals: Dilation and Erosion}

Morphology is a broad set of image processing operations that process images based on shapes. Morphological operations apply a structuring element to an input image, creating an output image of the same size. In a morphological operation, the value of each pixel in the output image is based on a comparison of the corresponding pixel in the input image with its neighbors. By choosing the size and shape of the neighborhood, you can construct a morphological operation that is sensitive to specific shapes in the input image.

The most basic morphological operations are dilation and erosion. Dilation adds pixels to the boundaries of objects in an image, while erosion removes pixels on object boundaries. The number of pixels added or removed from the objects in an image depends on the size and shape of the structuring element used to process the image. In the morphological dilation and erosion operations, the state of any given pixel in the output image is determined by applying a rule to the corresponding pixel and its neighbors in the input image. The rule used to process the pixels defines the operation as a dilation or an erosion. This table lists the rules for both dilation and erosion.

(a) Rules for Dilation and Erosion

\begin{tabular}{|l|l|}
\hline Operation & Rule \\
\hline Dilation & $\begin{array}{l}\text { The value of the output pixel is the maximum value of all the pixels in the input } \\
\text { pixel's neighborhood. In a binary image, if any of the pixels is set to the value }\end{array}$ \\
\hline
\end{tabular}


International Journal of Instrumentation and Control Systems (IJICS) Vol.2, No.1, January 2012

\begin{tabular}{|l|l|}
\hline Erosion & 1, the output pixel is set to 1. \\
\hline $\begin{array}{l}\text { The value of the output pixel is the minimum value of all the pixels in the input } \\
\text { pixel's neighborhood. In a binary image, if any of the pixels is set to } 0, \text { the } \\
\text { output pixel is set to } 0 .\end{array}$ \\
\hline
\end{tabular}

\section{(b) Combining Dilation and Erosion}

Dilation and erosion are often used in combination to implement image processing operations. For example, the definition of a morphological opening of an image is an erosion followed by a dilation, using the same structuring element for both operations. The related operation, morphological closing of an image, is the reverse: it consists of dilation followed by an erosion with the same structuring element. The following section uses imdilate and imerode to illustrate how to implement a morphological opening. Note, however, that the toolbox already includes the imopen function, which performs this processing. The toolbox includes functions that perform many common morphological operations.

\section{(c) Morphological Opening}

You can use morphological opening to remove small objects from an image while preserving the shape and size of larger objects in the image. For example, you can use the imopen function to remove all the circuit lines from the original circuit image, circbw.tif, creating an output image that contains only the rectangular shapes of the microchips. To morphologically open the image, perform these steps:

1. Read the image into the MATLAB workspace.

BW1 = imread('circbw.tif');

2. Create a structuring element.

$\mathrm{SE}=\operatorname{strel}($ 'rectangle',[40 30]);

The structuring element should be large enough to remove the lines when you erode the image, but not large enough to remove the rectangles. It should consist of all 1's, so it removes everything but large contiguous patches of foreground pixels.

3. Erode the image with the structuring element.

4. $\mathrm{BW} 2=\operatorname{imerode}(\mathrm{BW} 1, \mathrm{SE})$; imshow(BW2)

This removes all the lines, but also shrinks the rectangles.

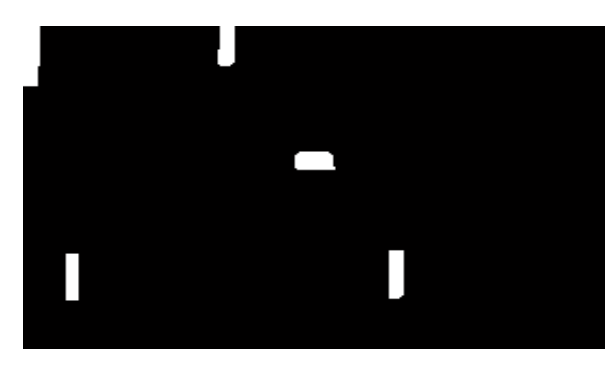

Fig 1: Morphologically open Rectangular microchips 
1. To restore the rectangles to their original sizes, dilate the eroded image using the same structuring element, SE.

2. $\mathrm{BW} 3=$ imdilate $(\mathrm{BW} 2, \mathrm{SE})$; imshow(BW3)

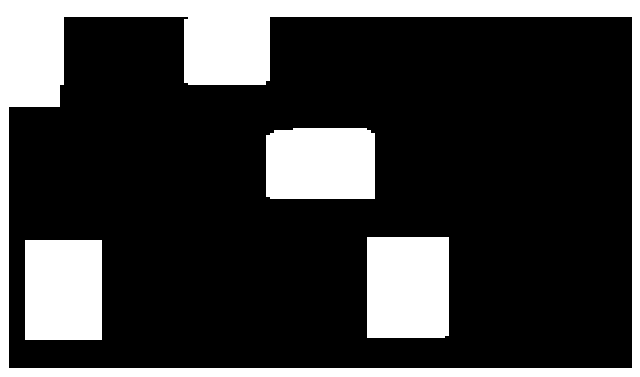

Fig 2: Dilation and Erosion of Rectangle

\section{Rode Obstacle Detection}

The process of detection on road obstacle is the process of identification and Calibration the target in the image [3] [4]. First, decrease the calculated amount by threshold segmentation and thinning method to the pre-processing image. Then, identify the target vehicle.

\subsection{Segmentation of Road Area}

In according to some features of the image, separate it into several regions, so that different parts will be significantly different. Threshold segmentation calculation, with the fast speed and stable performance, includes: (1) identification of segmentation threshold; (2) comparison and classification of segmentation threshold and pixel value. This paper extracts target from the image below, and makes a gray histogram statistic, and obtains the statistic and gray information from the histogram, and determines the basic location of the road. The basic process of obtaining the threshold value and detecting the road region including:

select the areas below the road, determine whether it involve both of the two categories gray scale value, namely pavements and markings. If not direct to step ;

select a initial threshold $T_{o}$, obtain the segmentation threshold of the two categories gray scale values by iteration method, and eliminate the impact of markings through the threshold value, then obtain an independent road gray scale;

calculate the range of gray scale $\mu_{u}$ and $\mu_{d}$ by parameter method;

Divide road region by $\mu_{u}$ and $\mu_{d}$.

\subsection{Binary Image Thinning}

After dividing the image in terms of the threshold value, then find out the basic lane areas. To reduce the calculated amount of obstacle detection, the image should be thinned. Image thinning 
refers to the operation of reducing the line width of the binary image as a pixel, then the processing line is known as a core wire. Thinning processing to simplify the calculation need some requests: replace the line width of core wire with a pixel; locate the core line at the center of the initial line; maintain the image continuity; provide the suspension of treatment if the processing time is too long. Define the mathematical morphology method as:

$S \oplus B=S \backslash(S \times B)$

Where the definition of set difference $S \backslash(S \times B)^{C}$

is $S \cap(S \times B)^{C},(S \times B)^{C}$ refers to the complementary set of initial image $S \times B$.

\subsection{Identification of Road Obstacle2}

The result of the identification of road obstacle in this study comes to "Yes" or "No", so it use the decision tree identification method in syntactic pattern recognition, with a series of judgment sentences dividing the pattern, making intuitive judgment without involving spatial alternation and measure problems. Moreover, it easy to be expressed in computer intuitive judgment and easy to achieve [5][6]. For the vehicle target in the image, obtain the location of the vehicle ahead, possibly by some certain criterion conditions to eliminate some elements, such as pedestrians, non-motor vehicles, etc. Establish the decision tree in terms of relevant factors in the identification processing: (1) within the road; (2) the difference between the targets connected domain gray and road area; (3) the grown form and range of the connected domain. After obtain the location of the vehicle ahead, calculate the practical location through the image, then find out the distance value of the vehicle ahead.

\section{Develop a Ranging Model}

After obtaining the location of obstacle in the image, input the data to the ranging model, and the distance of the vehicle ahead can be achieved. Therefore, it is important to develop the accuracy ranging model. The process of acquisition image by camera is the transform from 3-d Euler space to 2-d Euler space. To measure and calculate the practical data through the image data, it is the inverse process of the above one to some extent, and the model development is based on image transform.

\subsection{Image Transform Theory}

The geometric transform is a process of developing a Mapping function between the points in the initial image and the image after deformation. It is showed as follows:

$[x, y]=[X(u, v), Y(u, v)]$ or $\quad[u, v]=[U(x, y), V(x, y)]$

where $[u, v]$ refers to the co-ordinate of the output image pixel;

$[x, y]$ refers to the co-ordinate of the input image pixel.

$X 、 Y 、 U 、 V$ is the only mapping function that ascertain the spatial alternation, $X, Y$ define as forward mapping, which is mapping the input to output; and $U 、 V$ define as Reverse Mapping, 
which is mapping the output to the input. Generally show the space transformation with a $3 \times 3$ transformation matrix:

$$
\left[x^{\prime \prime}, y, w^{\prime}\right]=[u, v, w] T
$$

Where $T$ refers to transformation matrix; $x \square 、 y \square$ are a kind of manifestation mode of $x, y ; x$ 、 $y$ and $u 、 v$ are the coordinates before and after transformation respectively; and $w \square$ and $w$ are the homogeneous coordinate introduced for the treatment with three-dimensional matrix. The process of capturing Image by camera involves the image coordinate system and camera coordinate system and the world coordinate system. Figure I. shows the image coordinate system XC, YC. Each pixel coordinate is the numbers of rows and columns in the array. The axis $\mathrm{Z}$ of the camera coordinate system is vertical to the image plane, while the $X Y$ plane is parallel to the image plane. A datum coordinate system which expresses the location of camera and any other objects is known as the world coordinate system, expressed by $x, y, z$. The image plane is reflected by the focus, to prevent the use of mirror image with negative coordinate.

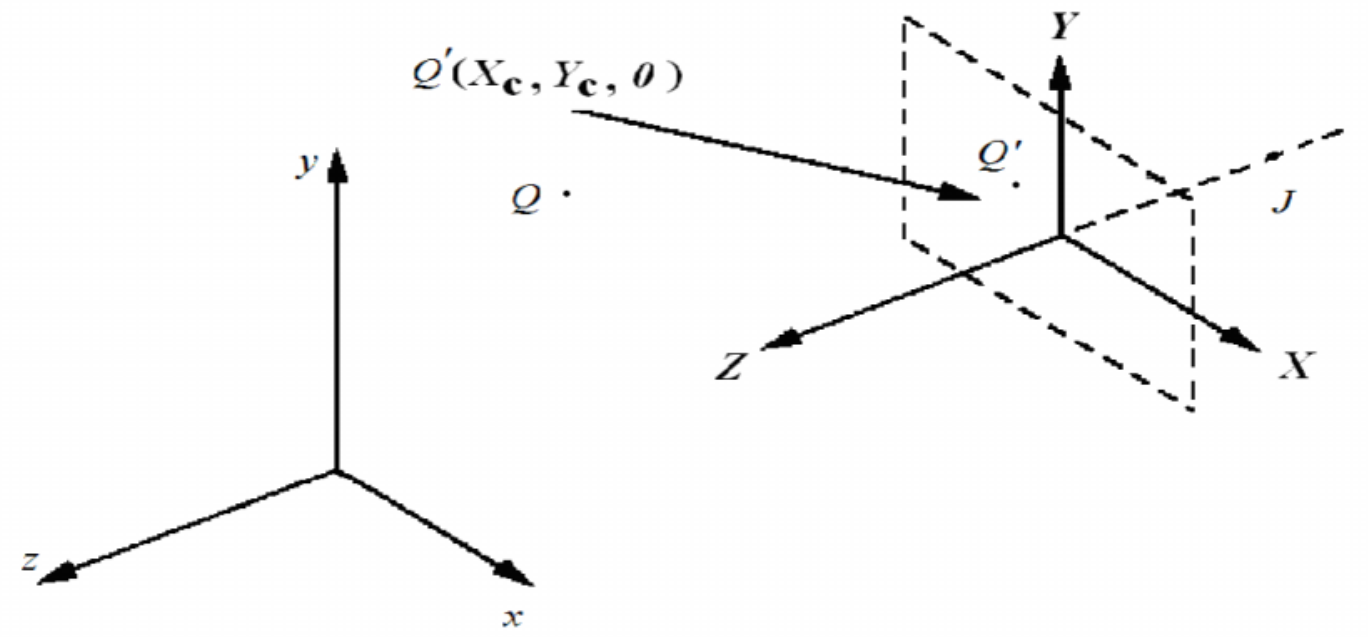

Figure 3 : Image, Camera and World Coordinate System

\subsection{Establish the Ranging Monocular Camera Model}

Given that the geometric transformation of the ranging monocular camera, if I refers to the three dimensional reality space, while $\mathrm{W}$ refers to the image space, the process of capturing image is transformation from I to W. After obtaining ordinate of the bottom of target vehicle in the image space, still need to gain conditions of the images collected and the assumptions for the image scene description. As shown in Figure 2, Camera location in the world coordinate system is showed by the positions of $1, d, h$; view direction is represented by the central axis of the camera with the standard shaft angle $\bar{Y}$ and $\bar{\theta}$; inclination of the camera aperture is $2 \alpha$; Camera Definition is $n \times n$. The above parameters can be obtained by measuring or indirectly calculating. $n \times n$ rays is from the camera to the outside space. The reality space angle of the ray which obtains point $(\mathrm{XC}, \mathrm{YC})$ in image space is determined by $\theta$ and $\Upsilon$. 
$(\mathrm{XC}, \mathrm{YC})$ in image space is determined by $\theta$ and $\Upsilon$.

$\Theta=(\bar{\theta}-\alpha)+Y_{c} \frac{2 \alpha}{n-1}$

Where $Y_{c}$ is the coordinates of the image space, $Y_{c} \square 0$,

$1,2, \ldots \ldots \ldots \ldots \ldots . ., \mathrm{n}-1$.

In addition, the ground point $\mathrm{P}$, the ground distance $\mathrm{dp}$ from the plane $\mathrm{S}$ to the camera can be calculated by the following formula:

$d_{p}=\frac{h}{\tan \theta}$

Taking these factors, get the formula for calculating the distance the vehicle ahead:

$$
d_{p}=\frac{h}{\tan \left[\left(\overline{\left.\theta-\alpha)+Y_{C} \frac{2 \alpha}{n-1}\right]}\right.\right.}
$$

The model has established the relationship between coordinates value $\mathrm{YC}$ in image space and the data of the real space plane $\mathrm{S}$ by the relationship among the ray angles. Thus, access to image pixel coordinates of the vehicle can calculate the vehicle's actual position in the plane S. As shown in Figure 2, if the central axis of the camera parallels with the world coordinate system zaxis, $\bar{\Upsilon}$ and $\bar{\theta}$ are zero, and the calculation process will be simplified:

$d_{p}=\frac{h}{\tan \left[\frac{2 \alpha Y_{C}}{n-1}-\alpha\right]}$

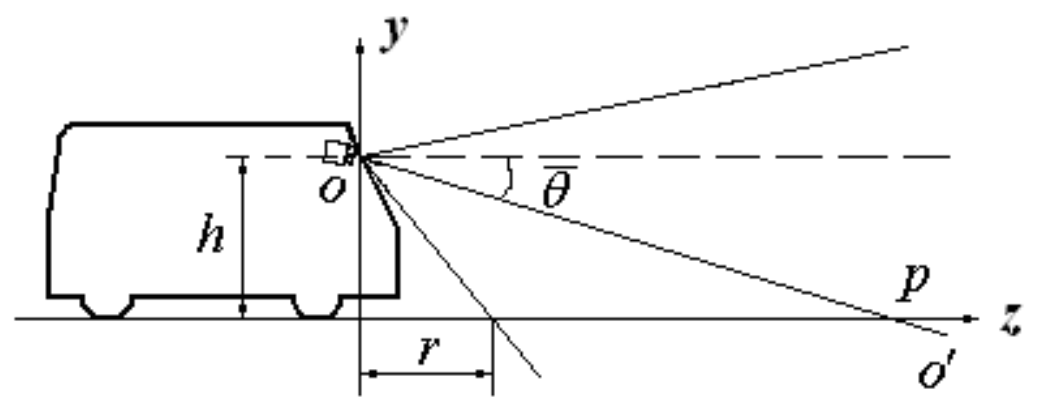

(a) Side View 


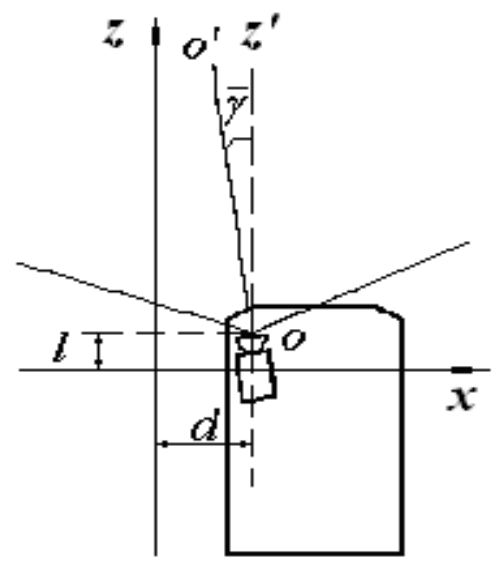

(b) Top View

Fig 4: Schematic diagram of camera parameters

\section{Implementation of Ranging Program}

Appropriate program is established based on ranging model. It has basic functions: achieve the image input, display, storage; and also need to integrate the image preprocessing, obstacle detection and distance algorithm for the vehicle ahead which were described in the paper before. This paper uses Visual $\mathrm{C}++$ to achieve ranging process based on digital image processing.

\subsection{Establishment of DIB Class}

Camera captured image is DIB (device independent bitmap) images, but there is no MFC class for the DIB bitmap. Such as the API function provided by the Win32 SDK is only invoked when using Visual $\mathrm{C}++$ to program, it not only in the processing is limited, but lose the advantages of object-oriented languages. Designing a series of function sets of dealing with DIB bitmap, and on this basis, to definite an exclusive class to deal with DIB bitmap can make procedures more efficient and targeted [7]. MFC's CObject class is the root class of most MFC classes. It offers many features and MFC derived classes have both a minimum level of member functions and data. Therefore, using it from API functions related to the Win32 SDK and based on a series of self-built DIB processing functions such as palette function, display function to establish CDib class with CObject class as a parent class.

\subsection{Establishment of Ranging Program}

In the establishment the basis of CDib class, the construction of function based on the CDib class can do edge enhancement, obstacle detection operations. Finally, to set up testing program under the AppWizard framework of $\mathrm{VC}++$ program and integrate these modules, then to establish testing program. In the establishment process of ranging program, it not only need to integrate the aforementioned image processing steps, but also to achieve the processing time calculation and information hintings and other functions. 


\section{Test and Analysis}

\subsection{Test Purpose and Test Scheme}

The test of measuring the leading vehicle distance is to capture the image of the vehicle ahead, which is based on computer ranging program, through the ranging program procedure to gain calculated value of the distance of the vehicle ahead, and to compare the calculated value with the actual distance between vehicles, then to draw program evaluation conclusions on the Ranging scheme. Straight sections of road are chosen as the actual test site.

The relevant test parameters are showed in Table I.

Table I. Basic parameters of the range Test

\begin{tabular}{|l|l|}
\hline Parameter & Parameter values \\
\hline $\bar{\gamma}$ & 0 \\
\hline $\bar{\theta}$ & 0 \\
\hline $\begin{array}{l}\text { Inclination of the camera } \\
\text { aperture } 2 \alpha\end{array}$ & 0.3236 \\
\hline Camera definition mxn & $2048 \times 1536$ \\
\hline $\begin{array}{l}\text { Distance from the camera } \\
\text { to the ground }\end{array}$ & 1.11 \\
\hline
\end{tabular}

\subsection{Results and analysis}

The test used five kinds distance of vehicle image to detect and then compared test results with the actual distance. The leading vehicle distance image preprocess recognition ranging process is shown in Figure 3. Measurement data, error and processing time are in Table II. It can prove that the method can more accurately detect the distance of the vehicle ahead by comparing the testdistance with actual distance. The time-consuming of image processing and calculation less than 1 second indicates that the method is simple, effective and more accurate to obtain the distance between vehicles ahead through a series of operation.

Table II. Test Data of Measuring the Leading Vehicle Distance

\begin{tabular}{|l|l|l|l|l|}
\hline No & $\begin{array}{l}\text { Actual } \\
\text { Distance } \\
(\mathrm{m})\end{array}$ & $\begin{array}{l}\text { Testing } \\
\text { Results } \\
(\mathrm{m})\end{array}$ & $\begin{array}{l}\text { Relative } \\
\text { Error } \\
(\%)\end{array}$ & $\begin{array}{l}\text { Processing } \\
\text { Time } \\
(100 \mathrm{~ms})\end{array}$ \\
\hline 1 & 20 & 20.45 & 2.25 & 4 \\
\hline 2 & 40 & 40.78 & 1.95 & 4 \\
\hline 3 & 60 & 64.95 & 8.25 & 3 \\
\hline 4 & 80 & 86.26 & 7.81 & 3 \\
\hline 5 & 100 & 92.31 & 7.39 & 4 \\
\hline
\end{tabular}


International Journal of Instrumentation and Control Systems (IJICS) Vol.2, No.1, January 2012

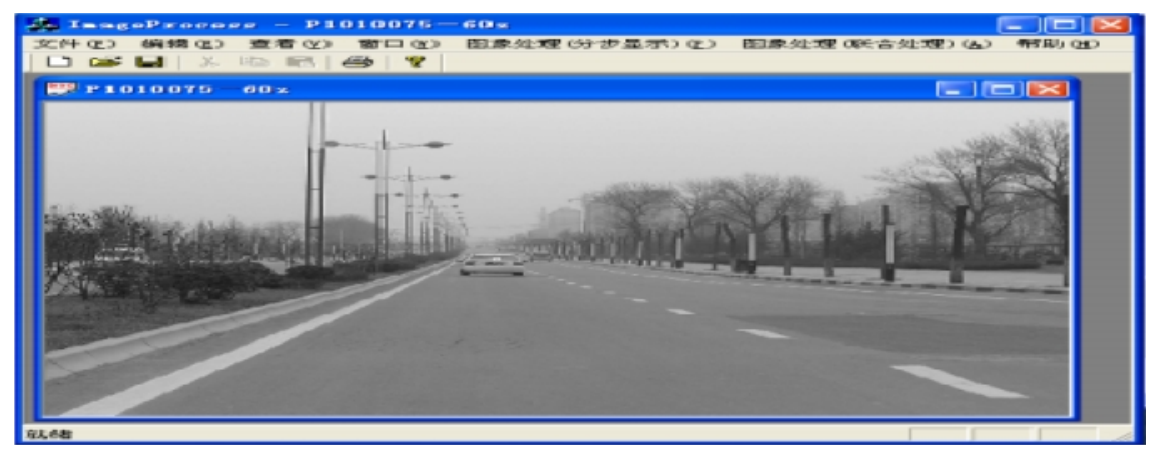

(a) Digital image collection

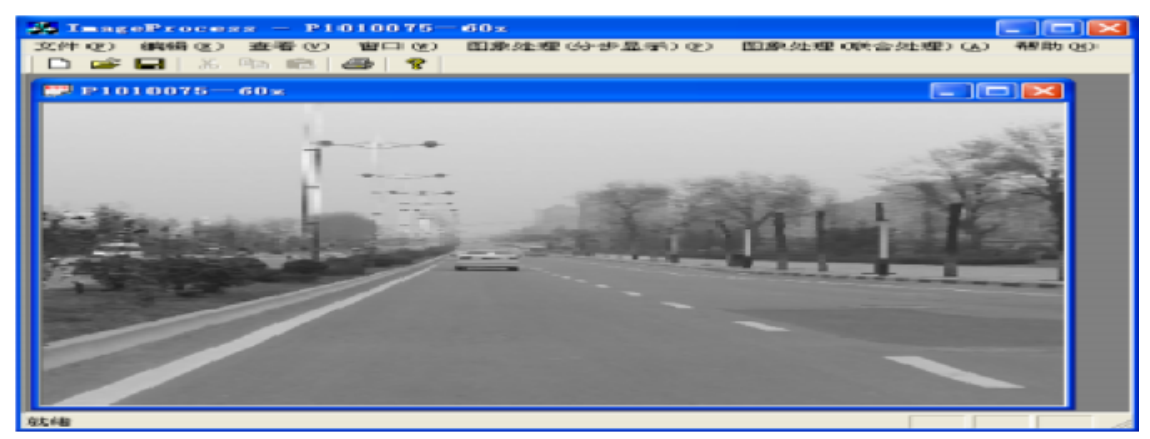

(b) Image Pre-processing

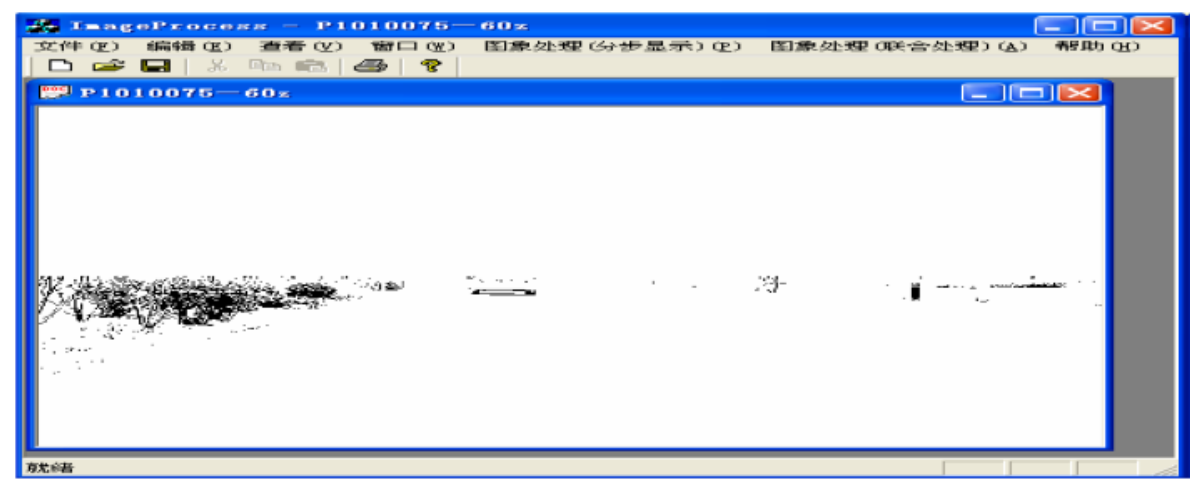

(c) Segmentation and Thinning 


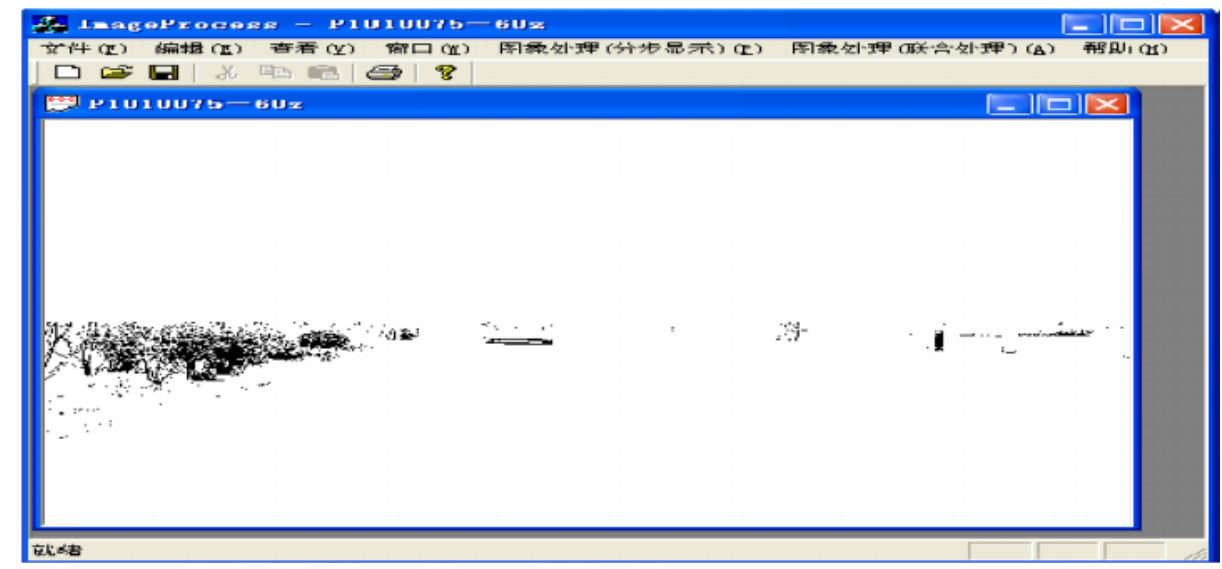

(d) Distance Measurement and Calculation

Fig 5 : Leading Vehicle distance image processing

\section{Conclusion}

This paper studied on a new method of ranging based on digital image processing which extracted the information and knowledge from the image, then obtained the leading vehicle distance through the use of morphological image preprocessing, threshold segmentation, decision tree with obstacles detection and the calculating model of inverse perspective mapping. By applying software $\mathrm{VC}++$ to write a test program, a ranging test was made. The test results proved that the method of measuring the leading vehicle distance is simple and effective. It can meet the requirement of intelligent vehicle technologies.

\section{References}

[1] Liu yan, Liu haoxue, Research on Lengthways Vehicle Distance Measurement System of Monocular Photograph Based on Computer Vision Journal of Highway and Transportation Research and Development. 2004, (9):103-106.

[2] Hong Zhang Lei Wang Ruiming Jia Junwei Li 2009.

[3] Ren Yong-gong;Yu Ge 2006.

[4] A. Blockey, Wang wuhong, Intelligent Vehicle. Beijing: China Communications Press, 2002, 14-37.

[5] Yang zhaohua, Pu zhaobang, Qi zhenqiang, Noise Removal from Image Data based on Mathematical Morphology and Genetic Optimization. Optical Technique.2004, (5):330-336.

[6] Romuald Aufrere, A model-driven approach for real-time road recognition. Machine Vision and Application, 2001, (13): 95-107.

[7] Xing yanchao, Tan zheng, Computer Vision-based Automatic Lane and Obstacle Detection. Computer Engineering and Applications. 2003, (6): 223-225.

[8] Garder W, Lawton D, Interactive mode based vehicle tracking. IEEE Transaction on Pattern Analysis and Machine Intelligence, 1996, (4): 1115-1121.

[9] Thomas Kalinke, A Texture-based Object Detection and an Adaptive Model-based Classification.Proceedings of the IEEE Intelligent Vehicles Symposium 1998, (10):143-148.

[10] LI Baozhong, HUANG Fengshan, state key Laboratory of Precision measuring technology and Instrument, China.

[11] P. LOVENITTI, William THOMPSON. Three-dimentional measurement using a single image, optical Engineering, 1996. 
International Journal of Instrumentation and Control Systems (IJICS) Vol.2, No.1, January 2012

[12] wei-Yen Wang, Department Applied Electronics Technology, National Taiwan Normal University, Taipei, Taiwan.

[13] Jinwei Yu, Jian Song, Xueyan Sun, College of Machinery, Weifang University Weifang, Shandong Province, P. R. China.

[14] Hugh Liu, Grantham Pang, Dept. of Electrical and Electronic Engineering, The University of Hong Kong, Pokfulam Road, Hong Kong. 